\title{
Diagnóstico de la cultura organizacional del programa educativo de Ingeniería Industrial de la Universidad Tecnológica de Tecamachalco
}

\section{Diagnosis of the organizational culture of the educational program of Industrial Engineering of the Universidad Tecnológica de Tecamachalco}

\author{
REYES-FERNÁNDEZ, Gabriela†, GONZÁLEZ-DÍAZ, Yolanda y AVELINO-ROSAS, Roberto \\ Universidad Tecnológica De Tecamachalco, Av. Universidad Tecnológica $N^{\circ} 1$, Col. Barrio la Villita, Tecamachalco, \\ Puebla, C.P 75483
}

ID $1^{\text {er }}$ Autor: Gabriela, Reyes-Fernández / ORC ID: 0000-0001-5556-3837, Researcher ID Thomson: S-5752-2018, CVU CONACYT ID: 500020

ID 1 ${ }^{\text {er }}$ Coautor: Yolanda, González-Díaz / ORC ID: 0000-0003-1132-4097, Researcher ID Thomson: S-5891-2018, CVU CONACYT ID: 947068

ID $2^{\text {do }}$ Coautor: Roberto, Avelino-Rosas / ORC ID: 0000-0002-1564-4302, Researcher ID Thomson: S-6538-2018, CVU CONACYT ID: 344859

DOI: $10.35429 /$ JOTE.2020.13.4.11.20

Recibido 03 de Septiembre, 2020; Aceptado 05 de Diciembre, 2020

\begin{abstract}
Resumen
El presente trabajo de investigación presenta un diagnóstico de la cultura organización del Programa educativo de Ingeniería Industrial de la Universidad Tecnológica de Tecamachalco, de acuerdo a los hallazgos encontrados y donde se pueda intervenir para establecer estrategias de mejora. Para llevar a cabo la investigación y con base en la revisión de literatura, se utilizó el método cuantitativo mediante el modelo Competing Values Framework como el más adecuado para diagnosticar la cultura organizacional en una institución educativa, tomando en consideración estudios realizados en México (García, Hernández, Vargas y Cuevas, diciembre 2012; Sánchez, 2017; Chuc, 2017) y que para su aplicación en un estudio de caso fue necesario determinar la validez y confiabilidad del instrumento. Para lograr entender cómo una organización actuará bajo diferentes circunstancias, se debe conocer qué esquemas de suposiciones básicas tiene, esto es, indagar en su cultura organizacional para conocer "valores compartidos, hábitos, usos y costumbres, códigos de conducta, políticas de trabajo, tradiciones y objetivos que se transmiten de una generación a otra." (Chiavenato, 2009, p. 120).
\end{abstract}

Diagnosis, Organizational Culture, Educational Institution

\begin{abstract}
This research work presents a diagnosis of the organizational culture of the Educational Program of Industrial Engineering of the Technological University of Tecamachalco, according to the findings found and where it can intervene to establish improvement strategies. To carry out the research and based on the literature review, the quantitative method was used through the Competing Values Framework Model as the most appropriate to diagnose the organizational culture in an educational institution, taking into consideration studies carried out in Mexico (García, Hernández, Vargas and Cuevas, December 2012; Sánchez, 2017; Chuc, 2017) and that for its application in a case study it was necessary to determine the validity and reliability of the instrument. In order to understand how an organization will act under different circumstances, it is necessary to know what basic assumptions it has, that is, to inquire into its organizational culture to know "shared values, habits, uses and customs, codes of conduct, work policies, traditions and objectives that are transmitted from one generation to another. " (Chiavenato, 2009, p. 120).
\end{abstract}

Diagnosis, Organizational Culture, Educational Institution

Citación: REYES-FERNÁNDEZ, Gabriela, GONZÁLEZ-DÍAZ, Yolanda y AVELINO-ROSAS, Roberto. Diagnóstico de la cultura organizacional del programa educativo de Ingeniería Industrial de la Universidad Tecnológica de Tecamachalco. Revista de Educación Técnica. 2020, 4-13: 11-20 


\section{Introducción}

Como es sabido estudiar a las organizaciones a través de su cultura permite conocer su esencia. Ésta esencia tal como lo menciona Dávila y Martínez (2009) está integrada por elementos que se necesitan identificar para comprender la vida de una organización. Al indagar sobre la cultura que predomina en las empresas en su forma más pura, permite también identificar su influencia en los procesos organizacionales. De acuerdo a Cameron y Quinn (2006), la cultura es un factor crucial en la eficacia a largo plazo de las organizaciones. Tales autores sugieren que los encargados de estudiar y gestionar la cultura organizacional deben ser capaces de medir las dimensiones clave de la misma y el desarrollo de destrezas para lograr el cambio.

El éxito organizacional y consecuentemente el desempeño se encuentra implícito y ampliamente vinculado de manera empírica con la cultura organizacional proporcionando el avance requerido para introducir cambios que modifiquen las formas tradicionales de realizar la gestión escolar (Muro, 2008; Hernández Sampieri, 2008).

El propósito de este trabajo es diagnosticar la cultura organizacional en sus 4 tipos: Clan, adhocracia, jerarquía y mercado; y poder determinar la cultura predominante del programa educativo de Ingeniería Industrial y poder proponer propuestas de mejora.

\section{Marco Teórico}

Una de las clasificaciones más empleadas para identificar la cultura organizacional predominante en una empresa, es la desarrollada por Cameron y Quinn, (1999) en la que proponen una metodología específica para el estudio de la cultura organizacional basada en el modelo conocido como Competing Values Framework (CVF). El propósito general de este modelo es diagnosticar, clasificar y facilitar el cambio de la cultura de una organización en particular, identificando cuatro grandes clases o tipos de cultura dominantes: A) Clan, B) Adhocracia, C) Jerárquica y D) Mercado. El Modelo de Valores en Competencia fue desarrollado por Cameron y Freeman (1991) y representa las presunciones básicas, orientaciones y valores de la cultura; aspectos considerados por varios autores como componentes de una cultura.
El modelo CVF muestra además si la organización tiene características predominantes en cuanto al grado de flexibilidad ante los cambios del entorno de parte de sus miembros; o por el contrario si la organización está predominantemente orientada a la estabilidad y control frente a los cambios.

Cultura de Clan. Se caracteriza por considerar a la empresa como una gran familia, de manera que el personal de la organización comparte muchos valores y objetivos, el estilo de dirección promueve el trabajo en equipo, el consenso y la participación. Como afirma Hellriegel y Slocum, (2009) la cultura de clan se caracteriza por la tradición, la lealtad, el compromiso personal, una extensa socialización, el trabajo en equipo, la autoadministración y la influencia social. Sus miembros reconocen una obligación que va más allá del sencillo intercambio de trabajos por un sueldo, entienden que la contribución a la organización (por ejemplo, horas trabajadas por semana) pueden exceder cualquier acuerdo contractual.

El compromiso de largo plazo del individuo con la organización (lealtad) es intercambiado por el compromiso de largo plazo de la organización con el individuo (seguridad). Puesto que las personas creen que la organización les dará un trato justo en materia de incrementos salariales, ascensos y otras formas de reconocimiento, se sienten responsables de sus actos ante la organización.

Una cultura de clan logra la unidad por medio de un largo y profundo proceso de socialización. Los miembros más viejos del clan sirven como mentores y modelos de función para los más nuevos.

El clan está consciente de la singularidad de su historia y frecuentemente documenta sus orígenes y celebra sus tradiciones con diversos ritos. Los miembros comparten una imagen de estilo y comportamiento de la organización. Las declaraciones y actos públicos refuerzan estos valores. En una cultura de clan, los integrantes comparten el orgullo de ser parte de la membresía. Tienen un fuerte sentido de identificación y reconocen su destino común en la organización. 
Cultura Adhocrática. La cultura Adhocrática se caracteriza por considerar a la empresa como una entidad dinámica y emprendedora, en la que se apuesta por nuevas ideas y por la asunción de riesgos, que valora la formalidad, las reglas y los procedimientos de operación establecidos como norma.

Las preocupaciones de largo plazo de una adhocracia son predecibles: eficiencia y estabilidad. Sus miembros aprecian mucho los productos y servicios al cliente estandarizado, las normas de comportamiento apoyan la formalidad sobre la informalidad. Los gerentes conciben sus funciones como buenos coordinadores, organizadores y vigilantes del cumplimiento de las reglas y normas escritas.

Las tareas, responsabilidades y autoridad están claramente definidas para todos los empleados, las reglas y procesos de la organización están contenidos en retos de las ciencias administrativas desde las economías emergentes: Evolución de sociedades, gruesos manuales y los empleados creen que su deber es conducirse "según el libro" y seguir los procedimientos legalistas. Por lo tanto, los flujos de energía corren de lo individual al equipo, dependiendo de la tarea que se encarga y del problema que se está abordando en ese momento; por lo tanto, los individuos en una adhocracia son a menudo los únicos tomadores de riesgo que anticipan y entienden el cambio (Cameron y Quinn,1999).

Cultura Jerárquica. La cultura jerárquica se caracteriza por estar jerarquizada, formalizada y estructurada, con normas y procedimientos previamente definidos para cualquier actividad, hace hincapié en un ambiente que es relativamente estable, donde las tareas y las funciones deben de ser integradas y coordinadas, la uniformidad en los productos y servicios se pueden mantener, y los trabajadores y el empleo están bajo control (Cameron y Quinn, 1999).

En esta cultura, el éxito se define por la incorporación en la toma de decisiones de forma clara por parte de la autoridad, las normas, los procedimientos normalizados, el control y mecanismos de rendición de cuentas, coordina y organiza la actividad para mantener una organización de liso funcionamiento.
La estabilidad, la previsibilidad, y la eficacia caracterizan las preocupaciones de largo plazo de esta organización (Cameron y Quinn, 1999).

Según, Hellriegel, Jackson y Slocum (2004) la cultura jerárquica se sitúa en un enfoque externo y flexible que crea un ambiente que alienta a asumir riesgos, a generar dinamismo y creatividad. Hay un compromiso con la experimentación, la innovación y el estar a la vanguardia. Esta cultura no sólo reacciona con rapidez a los cambios en el entorno, sino que los genera.

Cultura de Mercado. La cultura de mercado según Hellriegel, Jackson y Slocum (2004) es una cultura donde los valores y las normas reflejan la importancia de lograr objetivos mensurables y exigentes, en especial los que son financieros y se basan en el mercado (por ejemplo, crecimiento de las ventas, rentabilidad y participación de mercado).

En la organización predominan una competitividad muy intensa y una orientación a las ganancias. Su enfoque es externo y no ejerce mucha presión social informal en los integrantes de la organización, las interacciones de los superiores con los subordinados consisten en gran medida en negociar acuerdos de desempeño y recompensas y/o evaluar solicitudes de asignación de recursos. Una competitividad energética y la orientación hacia las ganancias prevalecen en toda organización.

La cultura de mercado se refiere a un tipo de organización que funciona como un mercado en sí mismo, esta organización se centra sobre todo en el ambiente externo, en transacciones con los proveedores, clientes, contratistas, concesionarios, uniones, reguladores, etc.

El mercado funciona sobre todo con intercambio monetario, competitividad y productividad; estas organizaciones son dependientes del mercado por lo que lo monitorean e identifican a quien lo controla (Cameron y Quinn, 1999).

Cada cultura organizacional refleja un perfil de atributos como lo son: el estilo de gestión, planes estratégicos, el clima, el sistema de recompensas, liderazgo y los valores básicos de la organización (Cameron y Quinn, 1999). 


\section{Pregunta de investigación}

¿Cuál es el tipo de cultura organizacional predominante en la Universidad Tecnológica de Tecamachalco, específicamente en el programa educativo de Ingeniería Industrial?

\section{Objetivo General}

Identificar y Diagnosticar la cultura organizacional de Ingeniería Industrial en la Universidad Tecnológica de Tecamachalco.

\section{Objetivos Específicos}

- Elegir un instrumento para diagnosticar el tipo de cultura organizacional del programa educativo de Ingeniería Industrial de la UTTECAM

- Aplicar encuesta a los docentes del programa educativo de Ingeniería Industrial de la UTTECAM

- Analizar Resultados de la encuesta

- Determinar el tipo de cultura organizacional del programa educativo de Ingeniería Industrial en la UTTECAM

- $\quad$ Establecer una propuesta de mejora del programa educativo mencionado

\section{Metodología a desarrollar}

El instrumento obtenido y utilizado para describir la cultura organizacional dominante en el programa educativo de Ingeniería Industrial de la Universidad Tecnológica de Tecamachalco, fue un formato de encuesta de una versión modificada del cuestionario OCAI desarrollado por (Chuc F. 2018).

\begin{tabular}{|l|l|}
\multicolumn{2}{|c|}{$\begin{array}{c}\text { Adaptación del instrumento para el diagnóstico de la } \\
\text { Cultura organizacional }\end{array}$} \\
\hline Término original & Adecuado a educación \\
\hline Organización & Institución (Dirección de carrera) \\
\hline Innovación & Mejora continua \\
\hline Toma de riesgos & Creatividad y emprendedurismo \\
\hline Resultados & Indicadores educativos \\
\hline Mercado & Alumnos \\
\hline Producción & Servicios educativos \\
\hline
\end{tabular}

Tabla 1

Fuente: Elaboración Propia
La siguiente herramienta cuantitativa que se utilizó es una encuesta para la evaluación para el estudio de la cultura organizacional basada en el modelo conocido como Competing Values Framework (CVF) se llevó acabo a 18 docentes de la carrera de Ingeniería Industrial del Universidad Tecnológica de Tecamachalco y tiene como objetivo definir la cultura organizacional predominante dentro de dicha Institución Educativa.

Instrucciones:

1. Responda las siguientes 6 preguntas (ítems) acerca de la Cultura Organizacional. Cada una de ellas contiene 4 alternativas (A, B, C, D).

2. No hay alternativas correctas o incorrectas para cada respuesta, por lo tanto, sea lo más sincero posible.

3. Distribuya 100 puntos por cada pregunta entre las cuatro, tres, dos o a una sola alternativa, dependiendo del grado de similitud que tenga la descripción con la realidad del Programa educativo de Ingeniería Industrial.

Observe que existe una columna de respuesta del instrumento etiquetada como "Ahora". Estas respuestas significan que está calificando a su institución educativa como está actualmente.

1. Características dominantes. El programa educativo es:

\begin{tabular}{|l|l|l|l|}
\hline \multicolumn{1}{|c|}{ Repuestas Actualmente } & $\begin{array}{c}\text { Preferido } \\
\text { (5 años) }\end{array}$ \\
\hline A & $\begin{array}{l}\text { Un lugar muy personal. Es como } \\
\text { una familia extensa. La gente } \\
\text { parece compartir mucho de ellos } \\
\text { mismos. }\end{array}$ & \\
\hline B & $\begin{array}{l}\text { Un lugar muy dinámico y de } \\
\text { mejora continua. La gente está } \\
\text { dispuesta a retar procesos. }\end{array}$ & \\
\hline C & $\begin{array}{l}\text { Muy orientada a los indicadores } \\
\text { educativos (como eficiencia } \\
\text { terminal, deserción, entre otros). }\end{array}$ & & \\
$\begin{array}{l}\text { Una preocupación importante es } \\
\text { hacer el trabajo bien hecho. La } \\
\text { gente es muy competitiva y } \\
\text { orientada hacia el logro. }\end{array}$ & \\
\hline D & $\begin{array}{l}\text { Un lugar con procesos y } \\
\text { procedimientos. } \\
\text { procedimientos fos } \\
\text { generalmente rigen lo que la } \\
\text { gente hace. }\end{array}$ & \\
\hline Total: & \\
\hline
\end{tabular}


2. Liderazgo organizacional. El Director de área generalmente se considera que ejemplifica:

\begin{tabular}{|l|l|l|l|}
\hline & \multicolumn{2}{|c|}{ Repuestas Actualmente } & $\begin{array}{l}\text { Preferido } \\
\text { (5 años) }\end{array}$ \\
\hline A & $\begin{array}{l}\text { La tutoría, la guía y la } \\
\text { enseñanza. }\end{array}$ & \\
\hline B & $\begin{array}{l}\text { El espíritu emprendedor, } \\
\text { la mejora continua y reta } \\
\text { procesos. }\end{array}$ & \\
\hline C & $\begin{array}{l}\text { Un enfoque sensato, } \\
\text { agresivo y orientado a los } \\
\text { indicadores educativos. }\end{array}$ & & \\
\hline D & $\begin{array}{l}\text { La coordinación, } \\
\text { organización o mejora la } \\
\text { eficiencia. }\end{array}$ & & \\
\hline & Total: & & \\
\hline
\end{tabular}

3. Gestión de los empleados. El estilo de gestión de la Dirección de carrera se caracteriza por:

\begin{tabular}{|c|c|c|c|}
\hline & Repuestas & $\begin{array}{l}\text { Actual } \\
\text { mente }\end{array}$ & $\begin{array}{l}\text { Preferido ( } 5 \\
\text { años) }\end{array}$ \\
\hline $\mathrm{A}$ & $\begin{array}{lll}\text { El trabajo en } & \text { equipo, } & \text { el } \\
\text { consenso } & y & \text { la } \\
\text { participación. } & & \\
\end{array}$ & & \\
\hline B & $\begin{array}{lr}\text { La toma de riesgos } \\
\text { individuales, } \\
\text { innovación, la libertad y la } \\
\text { singularidad. }\end{array}$ & & \\
\hline $\mathrm{C}$ & $\begin{array}{l}\text { La competencia, altas } \\
\text { exigencias y logros. }\end{array}$ & & \\
\hline $\mathrm{D}$ & $\begin{array}{l}\text { La seguridad del empleo, } \\
\text { la conformidad, la } \\
\text { previsibilidad y la } \\
\text { estabilidad en las } \\
\text { relaciones. }\end{array}$ & & \\
\hline & Total: & & \\
\hline
\end{tabular}

4. Unión de la institución educativa. Lo que mantiene unido al Programa educativo es:

\begin{tabular}{|c|c|c|c|}
\hline & Repuestas & Actualmente & $\begin{array}{c}\text { Preferido } \\
\text { (5 años) }\end{array}$ \\
\hline $\mathrm{A}$ & $\begin{array}{l}\text { La lealtad y la confianza } \\
\text { mutua. El compromiso } \\
\text { con el } \\
\text { educativo es alto. }\end{array}$ & & \\
\hline B & $\begin{array}{l}\text { El compromiso con la } \\
\text { innovación y el } \\
\text { desarrollo. Hay un énfasis } \\
\text { en estar a la vanguardia. }\end{array}$ & & \\
\hline $\bar{C}$ & $\begin{array}{l}\text { El énfasis sobre en el } \\
\text { logro y el cumplimiento } \\
\text { de metas. }\end{array}$ & & \\
\hline $\mathrm{D}$ & $\begin{array}{l}\text { Las reglas formales y } \\
\text { políticas. Mantener a la } \\
\text { institución educativa en } \\
\text { marcha es lo importante. }\end{array}$ & & \\
\hline & Total: & & \\
\hline
\end{tabular}

5. Énfasis estratégico. La dirección de carrera enfatiza:

\begin{tabular}{|c|c|c|c|}
\hline \multicolumn{2}{|c|}{ Repuestas } & \multirow{2}{*}{ Actualmente } & \multirow{2}{*}{$\begin{array}{c}\text { Preferido } \\
\text { (5 años) }\end{array}$} \\
\hline A & $\begin{array}{l}\text { El desarrollo humano. } \\
\text { La alta confianza, } \\
\text { apertura y participación } \\
\text { persisten. }\end{array}$ & & \\
\hline $\mathrm{B}$ & \begin{tabular}{|lrr} 
La adquisición & de \\
nuevos recursos y la \\
creación de & nuevos \\
retos. Se valoran & las \\
nuevas cosas y & la \\
búsqueda & & de \\
oportunidades. &
\end{tabular} & & \\
\hline $\mathrm{C}$ & $\begin{array}{l}\text { Las acciones y logros } \\
\text { competitivos. } \\
\text { objetivos de expansión } \\
\text { y el ganar espacios en } \\
\text { los medios educativos } \\
\text { son dominantes. } \\
\end{array}$ & & \\
\hline $\mathrm{D}$ & $\begin{array}{l}\text { La permanencia y la } \\
\text { estabilidad. } \\
\text { eficiencia, el control y } \\
\text { el buen funcionamiento } \\
\text { son importantes. } \\
\end{array}$ & & \\
\hline & Total: & & \\
\hline
\end{tabular}

6. Criterio de éxito. La dirección del Programa educativo define el éxito sobre la base de:

\begin{tabular}{|l|l|l|}
\hline \multicolumn{2}{|c|}{ Repuestas Actualmente } & \multicolumn{1}{|c|}{$\begin{array}{l}\text { Preferido } \\
\text { (5 años) }\end{array}$} \\
\hline A & $\begin{array}{l}\text { Desarrollo de los } \\
\text { recursos humanos, el } \\
\text { trabajo en equipo, } \\
\text { compromiso de los } \\
\text { empleados y la } \\
\text { preocupación por las } \\
\text { personas. }\end{array}$ & \\
\hline B & $\begin{array}{l}\text { El éxito sobre la base } \\
\text { de ser creativos, } \\
\text { emprendedor, con } \\
\text { propuestas nuevas. }\end{array}$ & \\
\hline C & $\begin{array}{l}\text { Ganar en el medio } \\
\text { educativo y superar la } \\
\text { competencia. rl El } \\
\text { liderazgo competitivo } \\
\text { en el medio educativo } \\
\text { es clave. }\end{array}$ & \\
\hline D & $\begin{array}{l}\text { La eficiencia. La } \\
\text { entrega confiable, el } \\
\text { cumplimiento de sus } \\
\text { tareas y la educación } \\
\text { a bajo costo son } \\
\text { fundamentales. }\end{array}$ & \\
\hline Total: & \\
\hline
\end{tabular}

Fuente: (Cameron y Quinn, 1999) 


\section{Resultados}

Los resultados obtenidos en la encuesta a 29 docentes del programa educativo de Ingeniería Industrial de la UTTECAM fueron los siguientes:

\section{Instrucciones:}

1. Responda las siguientes 6 preguntas (ítems) acerca de la Cultura Organizacional. Cada una de ellas contiene 4 alternativas (A, B, C, D).

2. No hay alternativas correctas $\mathrm{O}$ incorrectas para cada respuesta, por lo tanto, sea lo más sincero posible.

3. Distribuya 100 puntos por cada pregunta entre las cuatro, tres, dos o a una sola alternativa, dependiendo del grado de similitud que tenga la descripción con la realidad del Programa educativo de Ingeniería Industrial.

Observe que existe una columna de respuesta del instrumento etiquetada como "Ahora". Estas respuestas significan que está calificando a su institución educativa como está actualmente.

Nota: El resultado obtenido de las 18 personas encuestadas esta multiplicado por 100 que son los puntos de valor.

1. Características dominantes. El programa educativo es:

\begin{tabular}{|c|c|c|c|}
\hline & Repuestas & Actualmente & $\begin{array}{c}\text { Preferido } \\
\text { (5 años) }\end{array}$ \\
\hline A & $\begin{array}{l}\text { Un lugar muy personal. Es } \\
\text { como una familia extensa. La } \\
\text { gente parece compartir } \\
\text { mucho de ellos mismos. }\end{array}$ & 392 & 315 \\
\hline B & $\begin{array}{l}\text { Un lugar muy dinámico y de } \\
\text { mejora continua. La gente } \\
\text { está dispuesta a retar } \\
\text { procesos. }\end{array}$ & 487 & 487 \\
\hline $\mathrm{C}$ & $\begin{array}{l}\text { Muy orientada a los } \\
\text { indicadores educativos (como } \\
\text { eficiencia terminal, } \\
\text { deserción, entre otros). Una } \\
\text { preocupación importante es } \\
\text { hacer el trabajo bien hecho. } \\
\text { La gente es muy competitiva } \\
\text { y orientada hacia el logro. }\end{array}$ & 506 & 530 \\
\hline $\mathrm{D}$ & $\begin{array}{l}\text { Un lugar con procesos y } \\
\text { procedimientos. } \\
\text { procedimientos } r \text { Los } \\
\text { generalmente rigen lo que la } \\
\text { gente hace. }\end{array}$ & 415 & 468 \\
\hline & Total: & 1800 & 1800 \\
\hline
\end{tabular}

Fuente: Elaboración Propia

\section{Caracteristicas dominantes. El programa educativo es (ACTUALMENTE):}
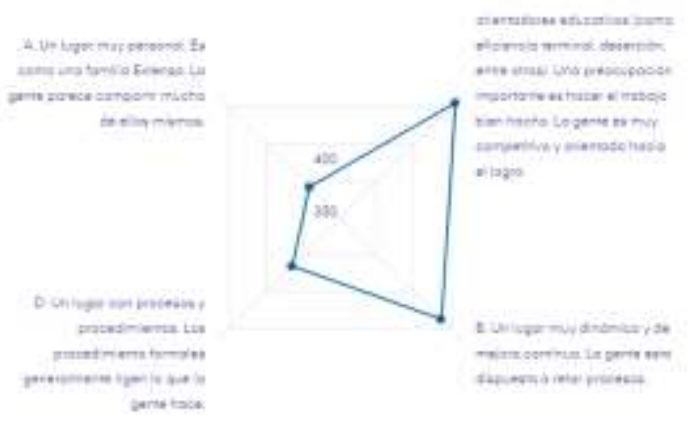

\section{Gráfico 1}

Fuente: Elaboración Propia

\section{Caracteristicas dominantes. El programa educativo (EN 5 AÑOS):}

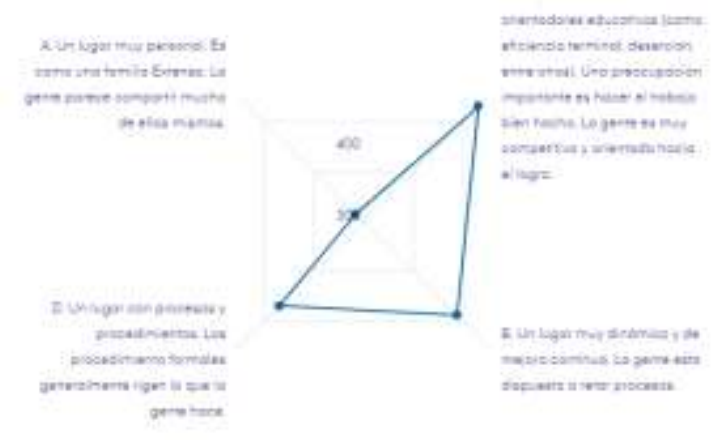

Gráfico 2

Fuente: Elaboración Propia

2. Liderazgo organizacional. El director de área generalmente se considera que ejemplifica:

\begin{tabular}{|l|l|r|r|}
\hline & \multicolumn{2}{|c|}{ Repuestas Actualmente } & $\begin{array}{c}\text { Preferido } \\
\text { (5 años) }\end{array}$ \\
\hline A & $\begin{array}{l}\text { La tutoría, la guía y la } \\
\text { enseñanza. }\end{array}$ & 404 & 480 \\
\hline B & $\begin{array}{l}\text { El espíritu emprendedor, } \\
\text { la mejora continua y reta } \\
\text { procesos. }\end{array}$ & 763 & 512 \\
\hline C & $\begin{array}{l}\text { Un enfoque sensato, } \\
\text { agresivo y orientado a los } \\
\text { indicadores educativos. }\end{array}$ & 319 & 432 \\
\hline D & $\begin{array}{l}\text { La coordinación, } \\
\text { organización o mejora la } \\
\text { eficiencia. }\end{array}$ & 314 & 376 \\
\hline & Total: & 1800 & 1800 \\
\hline
\end{tabular}

Fuente: Elaboración Propia 
3. Liderazgo organizacional. El director de área generalmente se considera que ejemplifica (ACTUAMENTE)

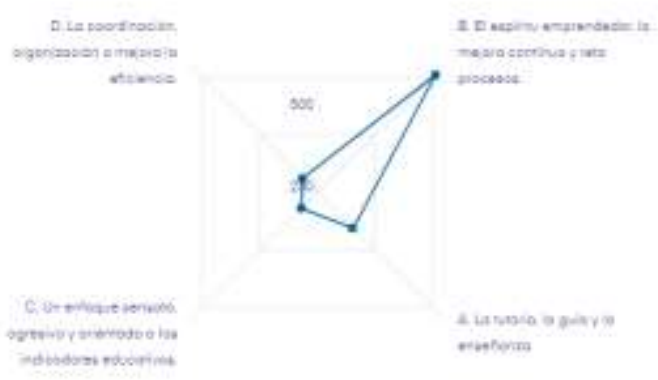

\section{Gráfico 3}

Fuente: Elaboración Propia

4. Liderazgo organizacional. El director de área generalmente se considera que ejemplifica (EN 5 AÑOS)

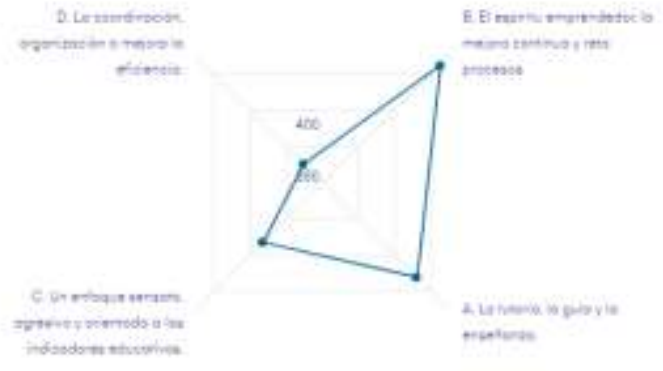

Gráfico 4

Fuente: Elaboración Propia

3. Gestión de los empleados. El estilo de gestión de la Dirección de carrera se caracteriza por:

\begin{tabular}{|c|c|c|c|}
\hline & Repuestas & Actualmente & $\begin{array}{l}\text { Preferido } \\
\text { (5 años) }\end{array}$ \\
\hline A & $\begin{array}{l}\text { El trabajo en equipo, el } \\
\text { consenso y la } \\
\text { participación. }\end{array}$ & 777 & 642 \\
\hline B & $\begin{array}{l}\text { La toma de riesgos } \\
\text { individuales, ra } \\
\text { innovación, la libertad y } \\
\text { la singularidad. }\end{array}$ & 251 & 513 \\
\hline $\mathrm{C}$ & $\begin{array}{l}\text { La competencia, altas } \\
\text { exigencias y logros. }\end{array}$ & 337 & 344 \\
\hline $\mathrm{D}$ & $\begin{array}{llr}\text { La seguridad } & \text { del } \\
\text { empleo, } & \text { la } \\
\text { conformidad, } & \text { la } \\
\text { previsibilidad y } & \text { la } \\
\text { estabilidad en las } \\
\text { relaciones. }\end{array}$ & 435 & 301 \\
\hline & TOTAL: & 1800 & 1800 \\
\hline
\end{tabular}

Fuente: Elaboración Propia 5. Gestión de los empleados. El estilo de gestión de
la dirección de la carrera se caracteriza por (ACTUALMENTE):

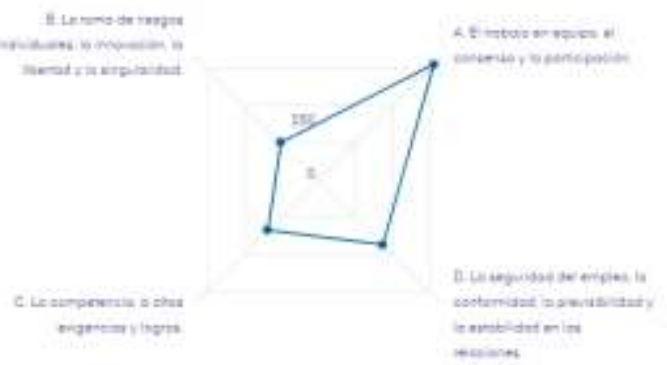

Gráfico 5

Fuente: Elaboración Propia

6. Gestión de los empleados. El estilo de gestión de la dirección de la carrera se caracteriza por (EN 5 AÑOS):

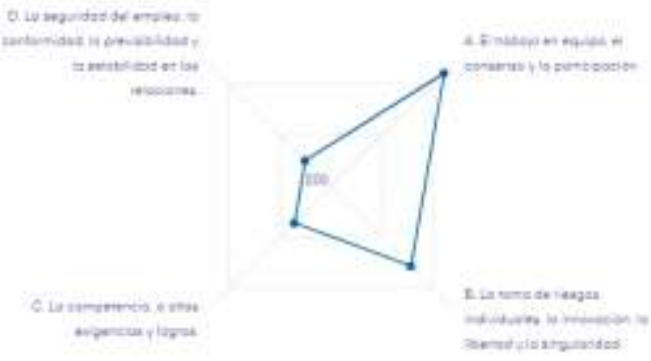

Gráfico 6

Fuente: Elaboración Propia

4. Unión de la institución educativa. Lo que mantiene unido al Programa educativo es:

\begin{tabular}{|c|c|c|c|}
\hline \multicolumn{2}{|r|}{ Repuestas } & Actualmente & $\begin{array}{c}\text { Preferido } \\
\text { (5 años) }\end{array}$ \\
\hline A & $\begin{array}{ll}\text { La lealtad y la } & \text { la } \\
\text { confianza mutua. } & \text { El } \\
\text { compromiso con el } \\
\text { Programa educativo es } \\
\text { alto. }\end{array}$ & 615 & 433 \\
\hline B & \begin{tabular}{l}
\multicolumn{3}{l}{ El compromiso con la } \\
innovación y \\
desarrollo. Hay un \\
énfasis en estar a la \\
vanguardia.
\end{tabular} & 309 & 464 \\
\hline $\mathrm{C}$ & $\begin{array}{lrr}\text { El énfasis } & \text { sobre } & \text { en } \\
\text { logro y y } & \text { el } \\
\text { cumplimiento } & \text { de } \\
\text { metas. } & \end{array}$ & 453 & 580 \\
\hline $\mathrm{D}$ & $\begin{array}{l}\text { Las reglas formales y } \\
\text { políticas. Mantener a la } \\
\text { institución educativa } \\
\text { en marcha es lo } \\
\text { importante. }\end{array}$ & 423 & 323 \\
\hline & TOTAL: & 1800 & 1800 \\
\hline
\end{tabular}

Fuente: Elaboración Propia 
7. Unión de la institución educativa. Lo que mantiene unido al programa educativo es (ACTUALMENTE):

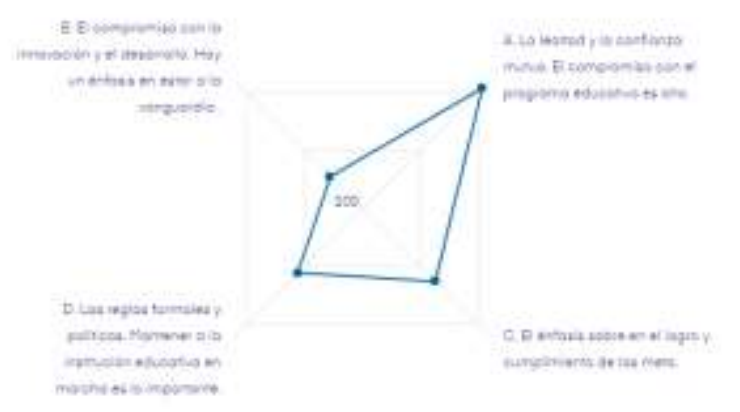

Gráfico 7

Fuente: Elaboración Propia

8. Unión de la institución educativa. Lo que mantiene unido al programa educativo es (EN 5 AN̄OS):

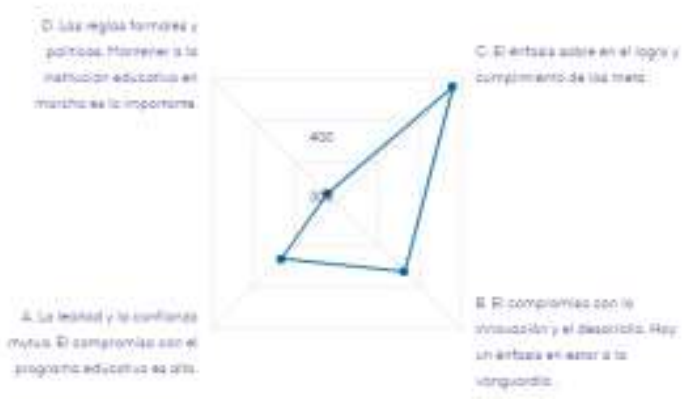

\section{Gráfico 8}

Fuente: Elaboración Propia

5. Énfasis estratégico. La dirección de carrera enfatiza:

\begin{tabular}{|c|c|c|c|}
\hline & Repuestas & Actualmente & $\begin{array}{c}\text { Preferido } \\
\text { (5 años) }\end{array}$ \\
\hline A & $\begin{array}{l}\text { El desarrollo humano. La } \\
\text { alta confianza, apertura y } \\
\text { participación persisten. }\end{array}$ & 359 & 604 \\
\hline B & $\begin{array}{l}\text { La adquisición de } \\
\text { nuevos recursos y la } \\
\text { creación de nuevos retos. } \\
\text { Se valoran las nuevas } \\
\text { cosas y la búsqueda de } \\
\text { oportunidades. }\end{array}$ & 649 & 458 \\
\hline $\mathrm{C}$ & $\begin{array}{l}\text { Las acciones y logros } \\
\text { competitivos. } \\
\text { objetivos de expansión y } \\
\text { el ganar espacios en los } \\
\text { medios educativos son } \\
\text { dominantes. }\end{array}$ & 392 & 456 \\
\hline $\mathrm{D}$ & $\begin{array}{l}\text { La permanencia y la } \\
\text { estabilidad. } \\
\text { eficiencia, el control y el } \\
\text { buen funcionamiento son } \\
\text { importantes. }\end{array}$ & 400 & 282 \\
\hline & Total: & 1800 & 1800 \\
\hline
\end{tabular}

Fuente: Elaboración Propia

ISSN-2523-2460

ECORFAN ${ }^{\circledR}$ Todos los derechos reservados

\section{9. Éntasis estratégico. La dirección de carrera enfatiza (ACTUAMENTE):}

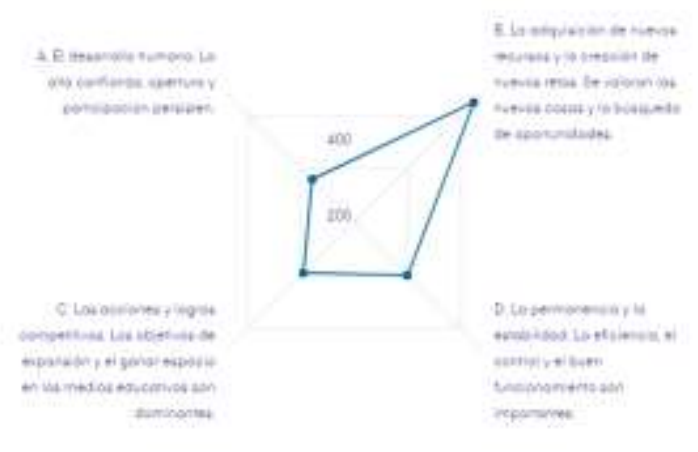

Gráfico 9

Fuente: Elaboración Propia

\section{0. Énfasis estratégico. La dirección de carrera enfatiza (EN 5 AÑOS):}

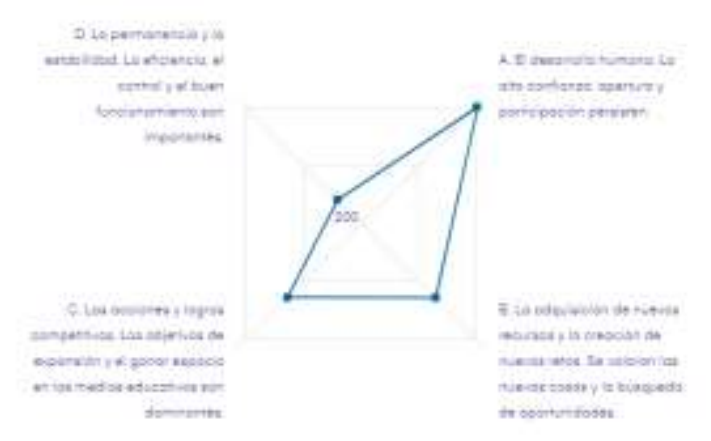

\section{Gráfico 10}

Fuente: Elaboración Propia

6. Criterio de éxito. La dirección del Programa educativo define el éxito sobre la base de:

\begin{tabular}{|c|c|c|c|}
\hline & Repuestas & Actualmente & $\begin{array}{c}\text { Preferido } \\
\text { (5 años) }\end{array}$ \\
\hline $\mathrm{A}$ & 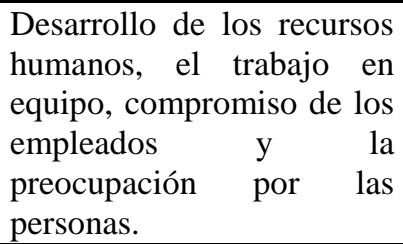 & 537 & 571 \\
\hline B & $\begin{array}{l}\text { El éxito sobre la base de } \\
\text { ser creativos, emprendedor, } \\
\text { con propuestas nuevas. }\end{array}$ & 357 & 486 \\
\hline $\mathrm{C}$ & $\begin{array}{l}\text { Ganar en el medio } \\
\text { educativo y superar la } \\
\text { competencia. El liderazgo } \\
\text { competitivo en el medio } \\
\text { educativo es clave. }\end{array}$ & 334 & 332 \\
\hline D & $\begin{array}{l}\text { La eficiencia. La entrega } \\
\text { confiable, el cumplimiento } \\
\text { de sus tareas y la educación } \\
\text { a bajo costo son } \\
\text { fundamentales. }\end{array}$ & 572 & 411 \\
\hline & Total: & 1800 & 1800 \\
\hline
\end{tabular}

Fuente: Elaboración Propia

REYES-FERNÁNDEZ，Gabriela，GONZÁLEZ-DÍAZ, Yolanda y AVELINO-ROSAS, Roberto. Diagnóstico de la cultura organizacional del programa educativo de Ingeniería Industrial de la Universidad Tecnológica de Tecamachalco. Revista de Educación Técnica. 2020 
11. Criterio de éxito. La dirección del programa educativo define el exito sobre la base de (ACTUALMENTE):

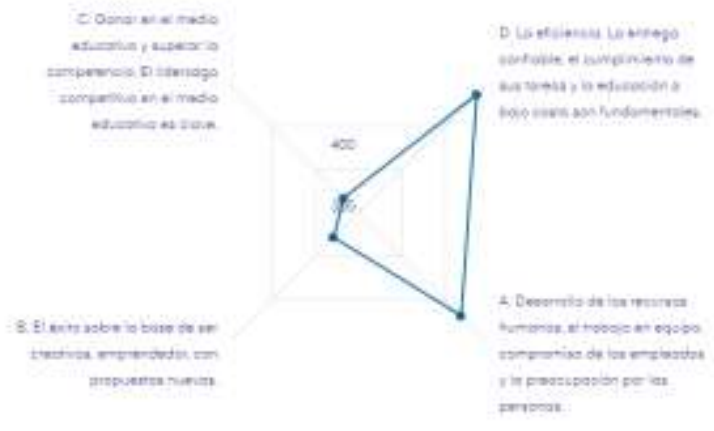

Gráfico 11

Fuente: Elaboración Propia

12. Criterio de éxito. La dirección del programa educativo define el éxito sobre la base de (EN 5 AÑOS):

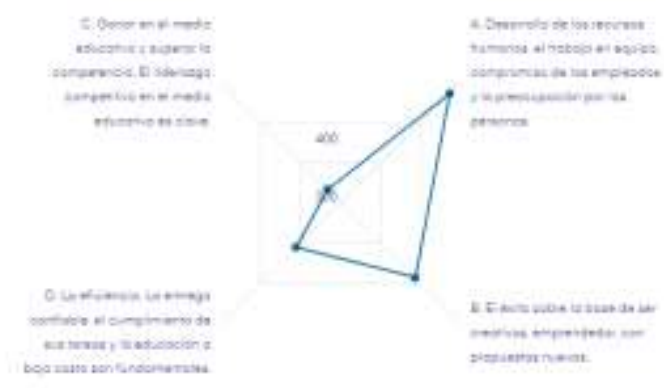

Gráfico 12

Fuente: Elaboración Propia

Promedio de resultados del cuestionario OCAI del P.E de Ingeniería industrial.

\begin{tabular}{|c|c|c|c|}
\hline & Cultura & Ahora & Preferido (5 años) \\
\hline $\mathrm{A}$ & Clan & 3084 & 3045 \\
\hline $\mathrm{B}$ & Adhocracia & 2816 & 2920 \\
\hline $\mathrm{C}$ & Mercado & 2341 & 2674 \\
\hline $\mathrm{D}$ & Jerarquía & 2559 & 2161 \\
\hline
\end{tabular}

Fuente: Elaboración Propia

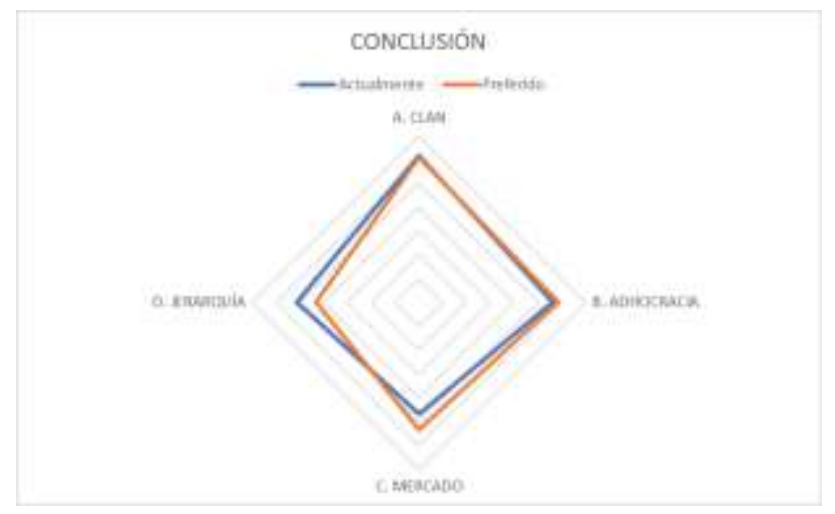

Gráfico 13

Fuente: Elaboración Propia
De acuerdo a los resultados de las encuesta se determinó que la cultura Clan es la predominante actualmente y se visualiza que siga predominando en 5 años dicho tipo de cultura tiene orientación colaborativa, el tipo de líder que maneja es mentor, facilitador y trabajo en equipo, los valores que predominan en esta cultura son el compromiso, comunicación, y desarrollo, la teoría de la efectividad en este tipo de cultura es el desarrollo humano y participación, lo que asegura una cultura muy adecuada al ser una organización del tipo Institución Educativa.

En segundo lugar, quedo el tipo de cultura de Adhocracia con una orientación más creativa, el tipo de líder es innovador, emprendedor y visionario, los valores que maneja son de innovación, transformación y agilidad y su teoría de la efectividad es innovación, visión y nuevos recursos. En tercer lugar quedó la cultura organizacional de jerarquía con orientación controlador, el tipo de líder coordinador, monitor y organizador, los valores son eficiencia, oportunidad, consistencia y uniformidad, la teoría de la efectividad de esta cultura es control y eficiencia de acuerdo a procesos establecidos. Finalmente, la cultura menos predominante es la de mercado que tiene los siguientes atributos una orientación de competencia, el tipo de líder estricto, competidor y productivo, los valores en esta cultura son estricto, competidor productivo, la teoría de la efectividad que se maneja aquí es centrado en la competencia y el cliente.

\section{Conclusiones}

Como se mencionó en los resultados se determinó que la cultura predominante en el programa de ingeniería industrial es la cultura del clan, aunque cabe recalcar que los resultados que se obtuvieron fueron muy equilibrados lo cuál habla de un equilibrio en la percepción de los empleados de dicha organización.

Se detectó que el estilo de la dirección del liderazgo y el comportamiento organizacional en el programa educativo de Ingeniería industrial posee actualmente un lugar de trabajo estructurado y formalizado, en donde se aprecia que la dirección suele ser bueno en coordinar y organizar. El éxito está definido en términos de la entrega confiable, la programación precisa y a bajo costo.

REYES-FERNÁNDEZ, Gabriela, GONZÁLEZ-DÍAZ, Yolanda y AVELINO-ROSAS, Roberto Diagnóstico de la cultura organizacional del programa educativo de Ingeniería Industrial de la Universidad Tecnológica de Tecamachalco. Revista de Educación Técnica. 2020 
Lo que el personal del programa educativo considera que la organización, debe ser un lugar dinámico, emprendedor y creativo. En donde el líder sea más innovador y tomador de riesgos. Y el éxito, está concebido como la generación de productos y de servicios nuevos y únicos. Por lo tanto, se trabajará con lo siguiente como propuesta de mejora:

Se pretende hacer una reestructuración organizacional en el programa educativo, que se verá reflejado en un cambio en el Organigrama de la carrera.

Hacer partícipes activos a los docentes para el monitoreo de los indicadores y los datos que corresponden a la media nacional, que toda la Universidad Tecnológica de Tecamachalco trabaja, como son:
a. Aprovechamiento 8.5 media TSU, Ingeniería 8.9.
b. Reprobación TSU 4.5, Ing 3\%, deserción 9.8 TSU.
c. Tasa de egreso Ing $4 \%$, tasa de egreso TSU generacional por cohorte 56, tasa de titulación por cohorte TSU 56.
d. Tasa de egreso Ing generacional por cohorte 77, tasa de titulación por cohorte Ing 72.

La realización de una investigación en la línea de la cultura organizacional y el liderazgo plantea la necesidad de realizar futuras investigaciones que consideren a mayor número de docente y a nivel institucional, incluyendo todas las carreras e inclusive las áreas administrativas de ésta para tener una tendencia mas clara a la cultura organizacional de toda la universidad.

Futuras investigaciones deberán contemplar variables como estructura de la organización y aspectos de comportamiento y de desempeño, además de considerar aspectos de la cultura propia del municipio y de ser posible del estado en que se realice.

\section{Referencias}

Andrade, Horacio (2011). Cambio o fuera. Dirigir en el siglo XXI. Estados Unidos de América: Palibrio.
Ávila, H. (2009). Análisis de la orientación al mercado y la cultura organizacional de las pequeñas empresas de la industria de artes gráficas local. Revista Internacional Administración \& Finanzas, 2 (1),19 -30.

Bell, F. (1995). Desarrollo organizacional (2a Ed.). Naucalpan, Edo. de México., México: Pearson/Prentice Hall.

Cameron, Kim S. y Quinn, Robert E. (2006). Diagnosing and Changing Organizational Culture. Based on the Competing Values Framework. United States of America: JosseyBass.

Meza, A. \& González, P.(2009). El Diagnóstico Organizacional; elementos, métodos y técnicas. Septiembre, 2019, de Mi espacio Sitio web: https://www.infosol.com.mx/espacio/Articulos/ Desde_la_Investigacion/El-DiagnosticoOrganizacionalelementos.html\#.XZEpCUZKi1s.

UTTECAM. (2020). Universidad Tecnológica de Tecamachalco, Universidad Tecnológica de Tecamachalco Sitio web: https://uttecam.edu.mx/eduma/ 